\title{
Charakterisierung der Quelldynamiken von smarten Hydrogelen mittels direkter optischer Erfassung
}

\author{
$\underline{\text { Guannan Mú }}{ }^{1}$, Klaudia Rückmann ${ }^{1}$, Julia Körner ${ }^{1}$ \\ 1 Institut für Grundlagen der Elektrotechnik und Messtechnik, \\ Leibniz Universität Hannover, Hannover, Deutschland \\ Kontakt: g.mu@geml.uni-hannover.de
}

\section{Motivation}

Stimuli-responsive (smarte) Hydrogele sind viskoelastische dreidimensionale Netzwerke aus hydrophilen Polymeren, welche die Fähigkeit besitzen, auf einen äußeren Parameter oder Stimulus (z. B. Ionenkonzentration, Licht oder $\mathrm{pH}$-Wert) mit einer Volumenänderung durch Wasseraufnahme bzw. abgabe zu reagieren [1,2]. Zusammen mit ihrer einfach zu erreichenden Bioverträglichkeit hat diese Art von Polymeren ein sehr großes Potential als Sensorelement im biomedizinischen Bereich. Einsatzmöglichkeiten reichen von passiven Anwendungen (z.B. Stützmatrix für Zellwachstum, Träger für Medikamente) bis hin zu Transducern für die Konzentration von Biomolekülen [3]. Insbesondere für letzteren Fall ist es bei der Entwicklung neuartiger anwendungsspezifischer Hydrogele notwendig, das dynamische Quellverhalten zu untersuchen und zu charakterisieren. Gängige Methoden wie Wiegemessungen und Rasterelektronenmikroskopie stoßen dabei an Grenzen, da diese z.B. kaum eine Echtzeitbeobachtung zulassen und in der Regel zudem (irreversible) Modifikationen der Proben erfordern. Deshalb ist es notwendig, neue Untersuchungsansätze zu entwickeln, die eine Echtzeitanalyse des dynamischen Quellverhaltens sowie die Beobachtung des statischen Verhaltens, insbesondere auch von kleinen Hydrogel-Proben, ermöglichen.

\section{Aufbau zur 3D optischen Charakterisie- rung von Hydrogel-Proben}

Hier wird dazu eine 3D optische Charakterisierungsmethode vorgestellt, die auf einer direkten Kameragestützten Beobachtung der Hydrogel-Probe in einer Fluidkammer basiert. Der Aufbau besteht aus einer 3D-gedruckten Kammer, die mit einem Glasboden (worauf das Hydrogel platziert wird) und einem Seitensichtfenster ausgestattet ist. An beiden Sichtfenstern ist jeweils eine Mikroskop-Kamera positioniert, mit welcher eine Bildaufnahme nach Benutzervorgaben mittels eines selbstgeschriebenen Python-Skriptes erfolgt. Die Fluidkammer ist zusätzlich mit mindestens einem Flüssigkeitseinlass und auslass für die Verwendung verschiedener Lösungen und einer oberen Öffnung ausgestattet, die für eine ausreichende Beleuchtung erforderlich ist (Abb. 1a).

Aus den aufgenommenen Bildern werden mit Hilfe eines weiteren selbstgeschriebenen Python-Skriptes die Querschnittsfläche und Dicke des Hydrogels in Abhängigkeit der Zeit extrahiert.

Die Flächenbestimmung basiert auf einer Kantenerkennung mittels Threshold (Schwellenwert)-Methode. Dabei werden die Pixel des aufgenommenen Bildes in eine Matrix übertragen und für jedes Element die Threshold-Bedingung nach dem RGBFarbschema überprüft. Liegt der Farbwert eines $\mathrm{Pi}$ xels über dem Schwellenwert, wird dieses zur Hydrogelfläche gezählt und im verarbeiteten Bild entsprechend farbig dargestellt, ansonsten wird dieses Pixel in Schwarz umgewandelt. Die Hydrogelfläche ergibt sich demzufolge aus der Anzahl farbiger $\mathrm{Pi}$ xel.

Wenn lediglich die relative Flächenänderung beim Quellen und Entquellen ausgewertet werden soll, kann direkt der Flächenwert in Pixel verwendet werden. Falls eine Umrechnung in $\mathrm{mm}^{2}$ oder $\mathrm{cm}^{2}$ erforderlich ist, muss entweder zum Anfang oder zum Ende einer Bilderserie eine Aufnahme mit einer Messskala (z.B. Lineal) erfolgen, mit welcher die Messwerte kalibriert werden können.

Die beschriebene Threshold-Methode kann für alle farbigen bzw. nicht-transparenten Hydrogele angewendet werden, wobei allerdings die Auswertebedingung entsprechend angepasst werden muss. Für eine rot gefärbte Probe wie in Abb. 1b lautet diese:

$$
R>T \cdot(1+B+G)
$$

wobei $R, G$ und $B$ die Farbwerte nach dem RGBSchema darstellen und $T$ den Threshold-Wert. Letzterer muss für die auszuwertende Bilderserie so gewählt werden, dass in allen Fällen mit demselben Schwellenwert eine korrekte Kantenerkennung erfolgt. Dies sollte insbesondere bei langen Serien beachtet werden, da hierbei z.B. Veränderungen der Beleuchtung oder geringfügige Variationen des Füllstandes der Kammer einen Einfluss haben können.

Für andere farbige Gele muss die Bedingung aus Gleichung (1) entsprechend modifiziert werden, so lautet diese z.B. für milchig-weiße Gele: 
$R+G+B>T$ mit $T>3 \cdot(255-x)$

Hierbei ist $x$ der festzulegende Schwellwert-Parameter.

Im Fall von transparenten Hydrogel-Proben kann die Threshold-Methode nicht verwendet werden. Hierbei kommt ein komplexerer Algorithmus zur Anwendung, wobei die Flächenbestimmung durch Umwandlung in ein Graustufenbild mit anschließender Verwendung eines Gauß- und eines Canny-Filters und ggf. Fehlerkorrekturen realisiert wird. Details hierzu finden sich in [4].

Aus den nach der Bildverarbeitung erhaltenen Kurven lassen sich direkt Rückschlüsse auf die dynamischen Eigenschaften wie z.B. Zeitkonstanten des Quellens und Entquellens, Hysterese und Überschwingen ziehen. Gleichzeitig können auch die statischen Eigenschaften, wie z.B. die maximale Volumenänderung im Gleichgewichtszustand, ermittelt werden.

Weiterhin erlauben die aufgenommenen Bilder eine dreidimensionale Rekonstruktion der betrachteten Probe und damit eine Analyse der Isotropie bzw. die Echtzeitbeobachtung der Morphologie während des Quellvorganges.

\section{Experimentelle Ergebnisse}

Die vorgestellte Charakterisierungsmethode wurde mit unterschiedlichen smarten Hydrogelen getestet, um Reproduzierbarkeit von Messungen, (Langzeit-)Stabilität, Sensitivität und Detektionslimit, sowie etwaige Herausforderungen und Einschränkungen zu eruieren. Abb. 1b zeigt beispielhaft originale und verarbeitete Bilder (Seitenansicht und Draufsicht) für ein $800 \mu \mathrm{m}$ dickes mit Gold-Nanopartikeln dotiertes Acrylamid-Hydrogel in einer Konzentration von 0,25x Phosphat-gepufferter Salzlösung (PBS). In Abb. 1c ist der aus einer Serie von 12.915 aufgenommenen Bildern extrahierte Zeitverlauf der Volumenänderung bei einem Konzentrationswechsel $1 \mathrm{x}-0,25 \mathrm{x}-1 \mathrm{x}$ PBS dargestellt. Dieser ergibt sich aus der Python-gestützten Auswertung der zeitlich synchronisierten Bildserien beider Kameras und der Schwellwert-Bedingung aus Gleichung (1).

\section{Zusammenfassung}

Mit dieser neuen Detektionsmethode ist es möglich, eine breite Palette von smarten Hydrogelen (chemische Zusammensetzung, Formen) in der gewünschten Lösungsumgebung sowohl hinsichtlich ihres dynamischen als auch stationären Verhaltens in einfacher und dennoch präziser Weise zu charakterisieren.

\section{Literatur}

[1] Bahram, M.; Mohseni, N.; Moghtader M. An Introduction to Hydrogels and Some Recent Applications; IntechOpen, 2016.

[2] A. Richter, G. Paschew, S. Klatt, J. Lienig, K. F. Arndt, H. P. Adler. Review on Hydrogel-based pH Sensors and Microsensors. Sensors 2008, 8(1), 561-581

[3] J. Tavakoli and Y. Tang. Hydrogel based sensors for biomedical applications: An updated review. Polymers, 9:264, 2017.

[4] K. Rückmann, G. Mu, J. Magda, F. Solzbacher, C. F. Reiche, J. Körner. A facile real-time optical characterization method for dynamic properties of smart hydrogels. In preparation, 2021.

(a)

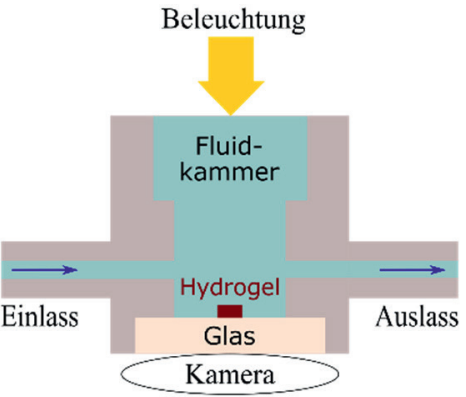

(b)

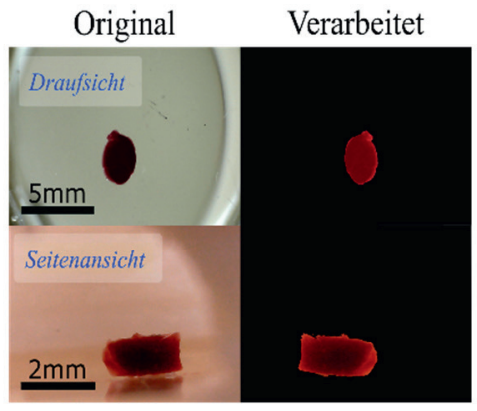

(c)

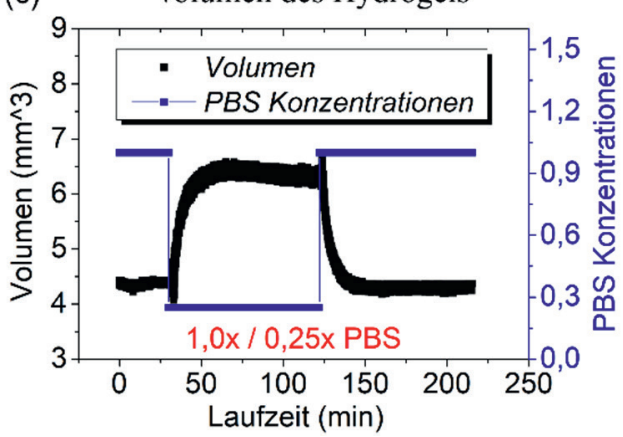

Abb. 1: (a) Aufbau zur optischen Charakterisierung. (b) Aufgenommenes (links) und verarbeitetes Bild (rechts) eines Acrylamid-basierten Hydrogels (Dicke $800 \mu \mathrm{m}$, Durchmesser $1 \mathrm{~mm}$ ) in 0,25x PBS-Lösung. (c) Dynamische Quellungsreaktion als tatsächliche Volumenänderung derselben Hydrogel-Probe in zwei verschiedenen PBS-Konzentrationen, die aus den verarbeiteten optischen Bildern extrahiert wurde. 